\title{
Simulations of thermally broadened H I Ly $\alpha$ absorption arising in the warm-hot intergalactic medium
}

\author{
P. Richter ${ }^{1}$, T. Fang ${ }^{2, \star}$, and G. L. Bryan ${ }^{3}$ \\ 1 Argelander-Institut für Astronomie ${ }^{\star \star}$, Universität Bonn, Auf dem Hügel 71, 53121 Bonn, Germany \\ e-mail: prichter@astro.uni-bonn.de \\ 2 Department of Astronomy, University of California at Berkeley, 601 Campbell Hall, Berkeley, CA 94720, USA \\ 3 Department of Physics, University of Oxford, Keble Road, Oxford OX13RH, UK
}

Received 21 November 2005 / Accepted 8 February 2006

ABSTRACT

Recent far-ultraviolet (FUV) absorption line measurements of low-redshift quasars have unveiled a population of intervening broad H I Ly $\alpha$ absorbers (BLAs) with large Doppler parameters $\left(b \geq 40 \mathrm{~km} \mathrm{~s}^{-1}\right)$. If the large width of these lines is dominated by thermal line broadening, the BLAs may trace highly-ionized gas in the warm-hot intergalactic medium (WHIM) in the temperature range $T \approx 10^{5}-10^{6} \mathrm{~K}$, a gas phase that is expected to contain a large fraction of the baryons at low redshift. In this paper we use a hydrodynamical simulation to study frequency, distribution, physical conditions, and baryon content of the BLAs at $z \approx 0$. From our simulated spectra we derive a number of BLAs per unit redshift of $(\mathrm{d} \mathcal{N} / \mathrm{d} z)_{\text {BLA }} \approx 38$ for H I absorbers with $\log \left(N\left(\mathrm{~cm}^{-2}\right) / b\left(\mathrm{~km} \mathrm{~s}^{-1}\right)\right) \geq$ $10.7, b \geq 40 \mathrm{~km} \mathrm{~s}^{-1}$, and total hydrogen column densities $N\left(\mathrm{H}_{\mathrm{II}}\right) \leq 10^{20.5} \mathrm{~cm}^{-2}$. The baryon content of these systems is $\Omega_{\mathrm{b}}(\mathrm{BLA})=$ $0.0121 h_{65}{ }^{-1}$, which represents $\sim 25$ percent of the total baryon budget in our simulation. Our results thus support the idea that BLAs represent a significant baryon reservoir at low redshift. BLAs predominantly trace shock-heated collisionally ionized WHIM gas at temperatures $\log T \approx 4.4-6.2$. About 27 percent of the BLAs in our simulation originate in the photoionized Ly $\alpha$ forest (log $T<4.3$ ) and their large line widths are determined by non-thermal broadening effects such as unresolved velocity structure and macroscopic turbulence. Our simulation implies that for a large-enough sample of BLAs in FUV spectra it is possible to obtain a reasonable approximation of the baryon content of these systems solely from the measured H I column densities and $b$ values.

Key words. methods: numerical - cosmology: miscellaneous - large-scale structure of Universe

\section{Introduction}

Cosmological simulations predict that a large fraction ( 30-40 percent) of the baryons in the local Universe exist in the form of hot $\left(T=10^{5}-10^{7} \mathrm{~K}\right)$, highly ionized intergalactic gas (e.g., Cen \& Ostriker 1999; Davé et al. 2001), commonly referred to as the warm-hot intergalactic medium (WHIM). The WHIM is believed to emerge from intergalactic gas that is shock-heated to high temperatures as the medium is collapsing under the action of gravity (Valageas et al. 2002). With current instrumentation, the most promising method to study the distribution and baryon content of the WHIM is to search for absorption lines from neutral hydrogen and highly-ionized heavy elements in far-ultraviolet and X-ray spectra of bright extragalactic background sources. Space-based observatories such as the Far Ultraviolet Spectroscopic Explorer (FUSE), the Hubble Space Telescope with its Space-Telescope Imaging Spectrograph (STIS), and the Chandra and XMM-Newton $\mathrm{X}$-ray observatories have been used to the study frequency and distribution of highly ionized oxygen (O VI, O VII, O VIII) in the WHIM toward a number of low-redshift background sources (e.g., Tripp et al. 2000; Savage et al. 2002; Fang et al. 2002; Shull \& Danforth 2005; Nicastro et al. 2005). FUV observations imply that the baryon content of O VI absorbers

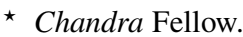

$\star \star$ Founded by merging of the Institut für Astrophysik und Extraterrestrische Forschung, the Sternwarte, and the Radioastronomisches Institut der Universität Bonn. (which are believed to trace the WHIM at temperatures around $T=3 \times 10^{5} \mathrm{~K}$ ) is $\Omega_{\mathrm{b}}(\mathrm{O} \mathrm{VI}) \geq 0.0022 h_{70}{ }^{-1}$ (Danforth \& Shull 2005; Sembach et al. 2004), but this estimate depends critically on the assumed oxygen abundance, which is not well constrained.

Apart from highly ionized oxygen, recent STIS observations by Richter et al. (2004, 2006) and Sembach et al. (2004) suggest that WHIM filaments can be traced by thermally broadened H I Ly $\alpha$ absorption (see also Williger et al. 2006). Although the vast majority of the hydrogen in the WHIM is ionized due to particle collisions and the ambient UV background radiation, a very small fraction $\left(<10^{-5}\right.$, typically) of the hydrogen is expected to be neutral if the gas is in ionization equilibrium. For WHIM filaments with total gas column densities $>10^{19} \mathrm{~cm}^{-2}$ the neutral hydrogen should give rise to weak Ly $\alpha$ absorption with H I column densities on the order of $\log N \approx 13$. Due to the high temperature of the gas $\left(T=10^{5}-10^{7} \mathrm{~K}\right)$ and the effect of thermal line broadening, the Ly $\alpha$ absorption is expected to be very broad and shallow with large Doppler parameters $\left(b \geq 40 \mathrm{~km} \mathrm{~s}^{-1}\right)$. About fifty of these broad Ly $\alpha$ absorbers (BLAs) have been identified in STIS low- $z$ QSO spectra so far (Richter et al. 2006), indicating that these systems represent a significant baryon reservoir at low redshift. The STIS measurements imply a number of BLAs per unit redshift of $(\mathrm{d} \mathcal{N} / \mathrm{d} z)_{\mathrm{BLA}} \geq 22$ for absorbers with $\log \left(N\left(\mathrm{~cm}^{-2}\right) / b\left(\mathrm{~km} \mathrm{~s}^{-1}\right)\right) \geq 11.3$ (detection limit for BLAs in STIS data with a signal-to-noise ratio of $\sim 18$ per $10 \mathrm{~km} \mathrm{~s}^{-1}$ wide pixel, hereafter referred to as $\left.(S / N)_{10}\right)$, and a baryon mass density of $\Omega_{\mathrm{b}}(\mathrm{BLA}) \geq 0.0027 h_{70}{ }^{-1}$. The advantage of using 
the BLAs for an estimate of $\Omega_{b}$ is that no assumption for the metallicity of the WHIM gas has to be made and therefore also low-metallicity environments can be detected. Large uncertainties for $\Omega_{\mathrm{b}}$ (BLA) arise, however, from the unknown contribution of non-thermal line broadening effects and the uncertain ionization fraction of the gas (see Richter et al. 2006).

In this paper we use artificial spectra generated from a cosmological simulation to study the frequency and baryon content of BLAs at low redshift. The aim of this paper is to test whether these simulations reproduce the observed spectral signatures of WHIM filaments (i.e., broad and shallow H I Ly $\alpha$ absorption) and to investigate the physical conditions in these systems. This paper is organized as follows: in Sect. 2 we explain the simulation method; in Sect. 3 we describe the spectral analysis of the artificial spectra; physical properties of the BLAs are discussed in Sect. 4; in Sect. 5 we estimate the baryon content of the BLAs in our simulation and compare the results with recent observations; in Sect. 6 we compare the relationship between BLAs and O vi absorbers; finally, we summarize our study in Sect. 7.

\section{Simulation method}

For our study we use the output of a numerical simulation that was part of an investigation of intervening O VI absorption arising in WHIM filaments (Fang \& Bryan 2001), and which is based on a grid-based adaptive mesh refinement method (Bryan 1996; Norman \& Bryan 1999). The generated cube is $20 \mathrm{~h}^{-1} \mathrm{Mpc}$ on a side and has a gas and dark matter mass resolution of $6 \times 10^{7}$ and $5 \times 10^{8} M_{\odot}$, respectively. The maximum resolution is $9.8 \mathrm{kpc}$ (smallest grid cell). The initial density field was drawn from an adiabatic cold dark matter power spectrum as approximated by the relation given in Eisenstein \& $\mathrm{Hu}$ (1998). The simulation is based on a cosmological model with $\Omega_{\mathrm{m}}=0.4, \Omega_{\mathrm{b}}=0.0473, \Omega_{\Lambda}=0.6$, and $h=0.65$ (in units of $\left.100 \mathrm{~km} \mathrm{~s}^{-1} \mathrm{Mpc}^{-1}\right)$. The inital redshift is $z=50$ and the simulation stops at $z=0.2$. Radiative heating and cooling of the gas is not included in the simulation, so that the gas temperature in the unshocked, low-density regions is significantly underestimated. For these regions we therefore approximate the temperature of the gas using the temperature-density relation $T=T_{0}(1+\delta)^{\gamma-1}$ found for the Ly $\alpha$ forest, where $T_{0} \approx 5000 \mathrm{~K}, \gamma \approx 1.4$ (e.g., Zhang et al. 1997) and $\delta$ is the overdensity of the gas. Note that previous studies indicate that radiative heating/cooling is not expected to significantly influence the evolution of the WHIM gas at low redshifts (see, e.g., Davé et al. 2001). However, we are planning to investigate this issue in more detail in a future paper. A density-dependent metallicity model was adopted, based on the results from Cen \& Ostriker (1999). More detailed information about the simulations can be found in Fang \& Bryan (2001) and Norman \& Bryan (1999) and references therein. The ionization state of the gas in the simulation has been modelled using the code CLOUDY (version 90.04; Ferland et al. 1998), which includes both collisional ionization and photoionization. A grid of temperatures $(\log T=3-7)$ and densities $\left(\log n_{\mathrm{H}}=-8\right.$ to -2$)$ is calculated from CLOUDY assuming an ionizing background spectrum at $z=0.2$ based on the model of Haardt \& Madau (1996) together with a mean specific intensity at the Lyman limit of $J_{v}=2 \times 10^{-23} \mathrm{erg} \mathrm{s}^{-1} \mathrm{~Hz}^{-1} \mathrm{sr}^{-1}$.

For the analysis of the simulated region we have drawn 3000 random lines of sight (LOS) through the simulated box and have created artificial spectra for $\mathrm{H} \mathrm{I}$ and O VI following the method described in Zhang et al. (1997). The total redshift path of these 3000 sightlines is $\Delta z=20.0$. The spectra then have been fitted using the automatted line fitting algorithm AutoVP
(Davé et al. 1997), which delivers column densities, $N$, and Doppler parameters, $b$, for the fitted spectral features.

\section{H I spectral analysis}

\subsection{BLA sample selection}

The fitting of the 3000 LOS with AutoVP results in the detection of 31,340 Ly $\alpha$ absorbers. The vast majority of these lines belong to the photoionized Ly $\alpha$ forest. 2671 of these lines have $b \geq 40 \mathrm{~km} \mathrm{~s}^{-1}$ and thus represent BLA candidates that may trace collisionally ionized gas at temperatures $T>10^{5} \mathrm{~K}$ arising in WHIM filaments. We have applied a number of selection criteria to identify the best BLAs candidates in our sample. First, we have excluded broad features that are part of complex multicomponent absorbers, as the determination of line centers, $b$ values, and column densities for these systems is afflicted with large systematic uncertainties. To exclude these crowded regions, we take into account only those BLA candidate lines whose nearest neighbouring absorption line is at least $2 \sigma$ away, where $\sigma$ is the standard deviation of a Gaussian-shaped line defined as:

$\sigma=\frac{F W H M}{2.354}=0.705 b$

In addition, we have excluded very weak BLA lines by constraining our BLA sample to H I absorbers that have an absorption strength of $\log \left(N\left(\mathrm{~cm}^{-2}\right) / b\left(\mathrm{~km} \mathrm{~s}^{-1}\right)\right) \geq 10.7$. This limit corresponds to a minimum absorption depth of $\sim 10$ percent. Note that lines with $\log (N / b)<10.7$ do not significantly contribute to the baryon content of the BLAs; moreover, they would clearly fall below the detection limit in FUV spectra that have signalto-noise ratios $(S / N)_{10} \leq 50$. After applying the above selection criteria we are left with a sample of 770 BLA candidate lines.

BLAs arising in the WHIM probably do not represent singlecomponent, isothermal gaseous structures, but rather are expected to be multi-phase systems with density substructure and internally varying ionization conditions. The method of using the BLAs to measure the baryon content of the WHIM is based on the idea that the shock-heated regions contain the dominant fraction of the baryons in these systems, and that these regions also determine the Ly $\alpha$ line width due to their high temperature and the resulting thermal line broadening. In an ideal case, one thus would expect to observe a single-component, Gaussianshaped broad absorption feature with a line-width that serves directly as a measure of the temperature of the gas (see also Sect. 3.4). However, if substantial substructure is present in a BLA, the thermal line-broadening is competing with the broadening of the absorption due to unresolved radial-velocity components. To distinguish between thermal broadening and nonthermal broadening it is therefore important to look for evidence of velocity-component structure in the BLA candidate lines (see also Richter et al. 2006). We have done a visual inspection of our 770 BLA candidates to evaluate the quality of the line fitting (as done by AutoVP) and to look for evidence for sub-component structure. 321 BLAs in our sample are almost perfectly Gaussian shaped and thus represent excellent candidates for thermally broadened WHIM absorbers. The remaining 449 BLAs show evidence for sub-component structure that has been ignored by the automated line fitting procedure. It could be that a large fraction of these broad systems may not be related to the WHIM but rather represent photoionized multi-component Ly $\alpha$ forest lines. Note that in real FUV spectra with $S / N$ ratios $(S / N)_{10}<50$ these multi-component systems would not be distinguishable from the 

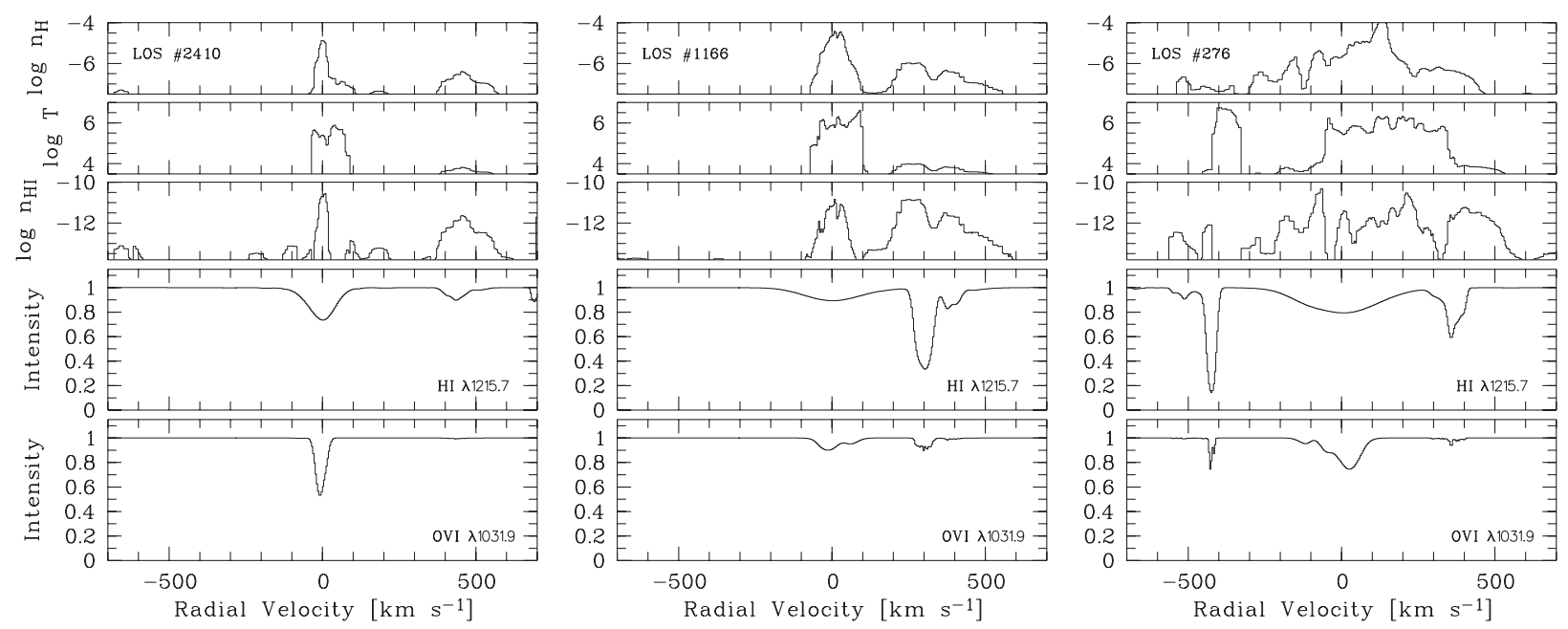

Fig. 1. Three examples for BLA absorbers in our simulated spectra are shown. The panels show the logarithmic total hydrogen volume density, gas temperature, neutral hydrogen volume density, and normalized intensity for H I Ly $\alpha$ and O VI $\lambda 1031.9$ absorption as a function of the radial velocity along each sightline. Densities $n$ and temperatures $T$ are in units $\mathrm{cm}^{-3}$ and K, respectively.

single-component cases. To compare our results with observational data it is therefore important to assess the properties of all BLA candidate lines in our sample. For the following analysis we distinguish between the high-quality BLA sample (321 absorbers) and the total BLA sample (770 absorbers).

Examples for BLAs along different lines-of-sight (LOS) in our simulated spectra are presented in Fig. 1, where we have plotted the total hydrogen volume density, the gas temperature, the neutral hydrogen volume density, the normalized H I Ly $\alpha \lambda 1215.7$ intensity, and the normalized intensity of the O VI $\lambda 1031.9$ line as a function of the restframe velocity for three BLAs from the high-quality sample. As clearly visible, the H I Ly $\alpha$ absorption in these systems is broad and symmetric. The left example (LOS 2410) shows a WHIM BLA with $b \approx$ $59 \mathrm{~km} \mathrm{~s}^{-1}$ and $\log N(\mathrm{HI})=13.37$; the density and temperature distribution is very simple in this system. The width of this BLA line is governed mainly by thermal broadening of the WHIM gas that has $\log T \approx 5.3$. The middle panel (LOS 1166) shows a thermally-broadened BLA with $b \approx 129 \mathrm{~km} \mathrm{~s}^{-1}$ and $\log N(\mathrm{HI})=$ 13.28. Also this system has a simple single-component structure, but the WHIM temperature is with $\log T \approx 5.9$ somewhat higher than in the left example, thus resulting in an H I Ly $\alpha$ line that is broader (and shallower). The right panel (LOS 276) shows an extremely broad BLA system with $b \approx 165 \mathrm{~km} \mathrm{~s}^{-1}$ and $\log N(\mathrm{HI})=13.70$. Here, the BLA system is characterized by a complex density distribution that spans several hundred $\mathrm{km} \mathrm{s}^{-1}$ (see upper panel). The BLA has a high temperature of $\log T \approx 6.0$, but in this case, the extremely large width of the line is not dominated by thermal broadening but by the complex velocity structure. The shape of the H I Ly $\alpha$ absorption slightly deviates from a perfect Gaussian, indicating the presence of velocity sub-components in this line. Such small asymmetries would remain unnoticed in real spectra where the $S / N$ is limited.

\subsection{BLA number density}

Figure 2 shows the cumulative number of BLAs per unit redshift, $\log (\mathrm{d} \mathcal{N} / \mathrm{d} z)$, plotted against the BLA absorption strength, $\log (N / b)$. The total sample (770 systems) is shown in gray, the high-quality sample (321) is plotted in black. For $b \geq$ $40 \mathrm{~km} \mathrm{~s}^{-1}$ and $\log (N / b) \geq 10.7$ our simulation predicts that $(\mathrm{d} \mathcal{N} / \mathrm{d} z)_{\mathrm{BLA}} \approx 39$ (total sample) and $(\mathrm{d} \mathcal{N} / \mathrm{d} z)_{\mathrm{BLA}} \approx 16$

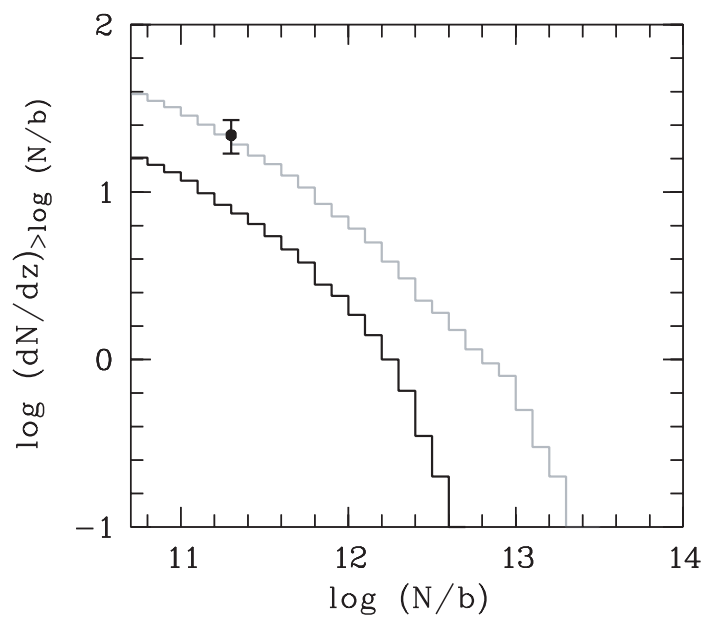

Fig. 2. Cumulative number of BLAs per unit redshift, $\log (\mathrm{d} \mathcal{N} / \mathrm{d} z)_{\mathrm{BLA}}$, as a function of the absorption strength, $\log \left(N\left(\mathrm{~cm}^{-2}\right) / b\left(\mathrm{~km} \mathrm{~s}^{-1}\right)\right)$. The total sample is shown in gray, the high-quality sample is plotted in black. The single data point indicates the value for $(\mathrm{d} \mathcal{N} / \mathrm{d} z)_{\text {BLA }}$ from FUV absorption line measurements with STIS (Richter et al. 2006).

(high-quality sample). For $\log (N / b) \geq 11.3$ the BLA number density in the total sample reduces to $(\mathrm{d} \mathcal{N} / \mathrm{d} z)_{\mathrm{BLA}} \approx 19$. This is very similar to the value obtained by recent STIS observations of BLAs towards low-redshift quasars $\left((\mathrm{d} \mathcal{N} / \mathrm{d} z)_{\mathrm{BLA}}=22\right.$ for reliably detected systems; Richter et al. 2006). The value for $(\mathrm{d} \mathcal{N} / \mathrm{d} z)_{\text {BLA }}$ from the STIS measurements is indicated in Fig. 2.

\subsection{HI column densities}

Figure 3 shows the distribution of logarithmic H I column densities and logarithmic $\mathrm{H}$ I $b$ values, as obtained from the profile fitting of the BLA candidate lines. The total sample is shown in gray, the high-quality sample is shown in black. The H I column density distribution (Fig. 3, upper panel) shows that the majority of the BLA systems ( $~ 92$ percent) have relatively low H I column densities with $\log N(\mathrm{HI}) \leq 14$ and a peak near $\log N(\mathrm{HI}) \approx 13$. Note that for column densities lower than that our sample is incomplete due to the exclusion of systems with $\log (N / b)<10.7$ (see Sect. 3.1). The true H I column density 

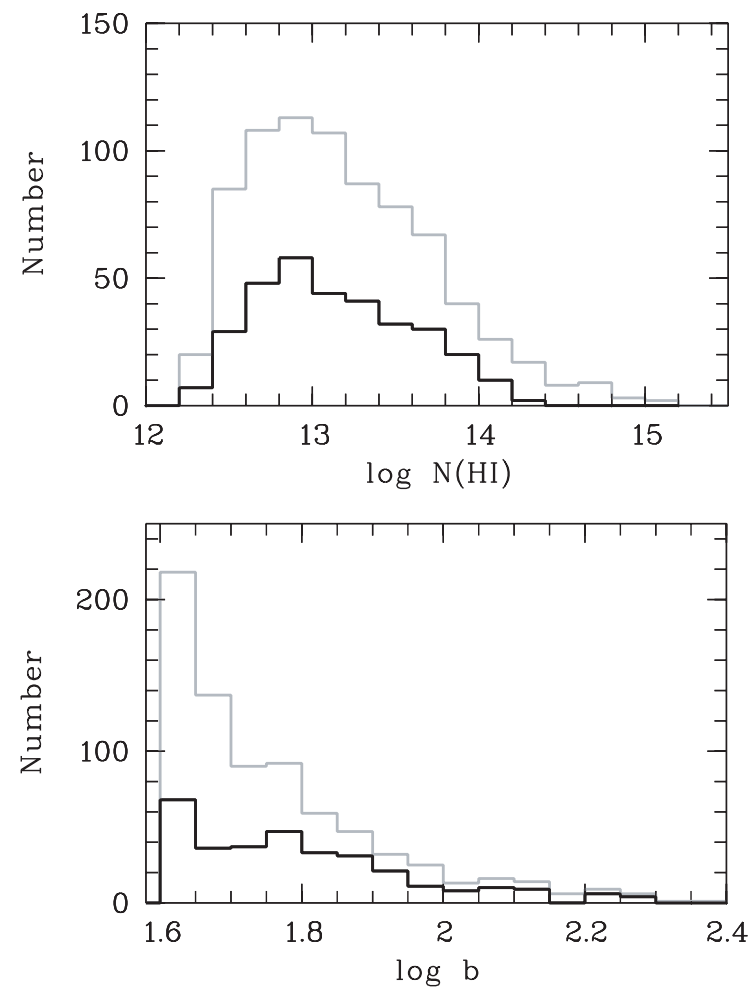

Fig. 3. H I column density and $b$ value distribution resulting from the Voigt fit of the BLA lines in the simulation. The gray line shows the distribution of the total sample (770 systems), the black line shows the distribution for the restricted sample (321 systems). Column densities are in units $\mathrm{cm}^{-2}, b$ values are in units $\mathrm{km} \mathrm{s}^{-1}$.

distribution of the BLAs most likely further rises towards lower column densities. The distribution of BLA H I column densities in our simulation is qualitatively very similar to the one observed in STIS spectra of low-z quasars (Richter et al. 2006; their Fig. 5).

\subsection{HI b values}

The BLA $b$ value distribution is plotted in the lower panel of Fig. 3. Some BLAs in our simulation have $b$ values larger than $200 \mathrm{~km} \mathrm{~s}^{-1}$, but most of the systems have relatively moderate line widths with $b$ values ranging from 40 to $100 \mathrm{~km} \mathrm{~s}^{-1}$ $(\log b=1.6-2.0)$. The $b$ values of the BLAs are assumed to be composed of a thermal component, $b_{\mathrm{th}}$, and a non-thermal component, $b_{\text {non-th }}$, in the way that

$b=\sqrt{b_{\mathrm{th}}^{2}+b_{\text {non-th }}^{2}}$.

The non-thermal component may include processes like macroscopic turbulence, unresolved velocity-components, and others (see Richter et al. 2006 for a detailed discussion). The contribution of the thermal component to $b$ depends on the gas temperature:

$b_{\mathrm{th}}=\sqrt{\frac{2 k T}{m}} \approx 0.129 \sqrt{\frac{T}{A}} \mathrm{~km} \mathrm{~s}^{-1}$

where $k$ is the Boltzmann constant, $m$ is the particle mass, and $A$ is the atomic weight. For the shock-heated WHIM gas with $\log T \geq 5$ one thus expects $b_{\text {th }} \geq 40 \mathrm{~km} \mathrm{~s}^{-1}$ for hydrogen. The non-thermal broadening mechanisms are expected to contribute to some degree to the total $b$ values in WHIM absorbers (see
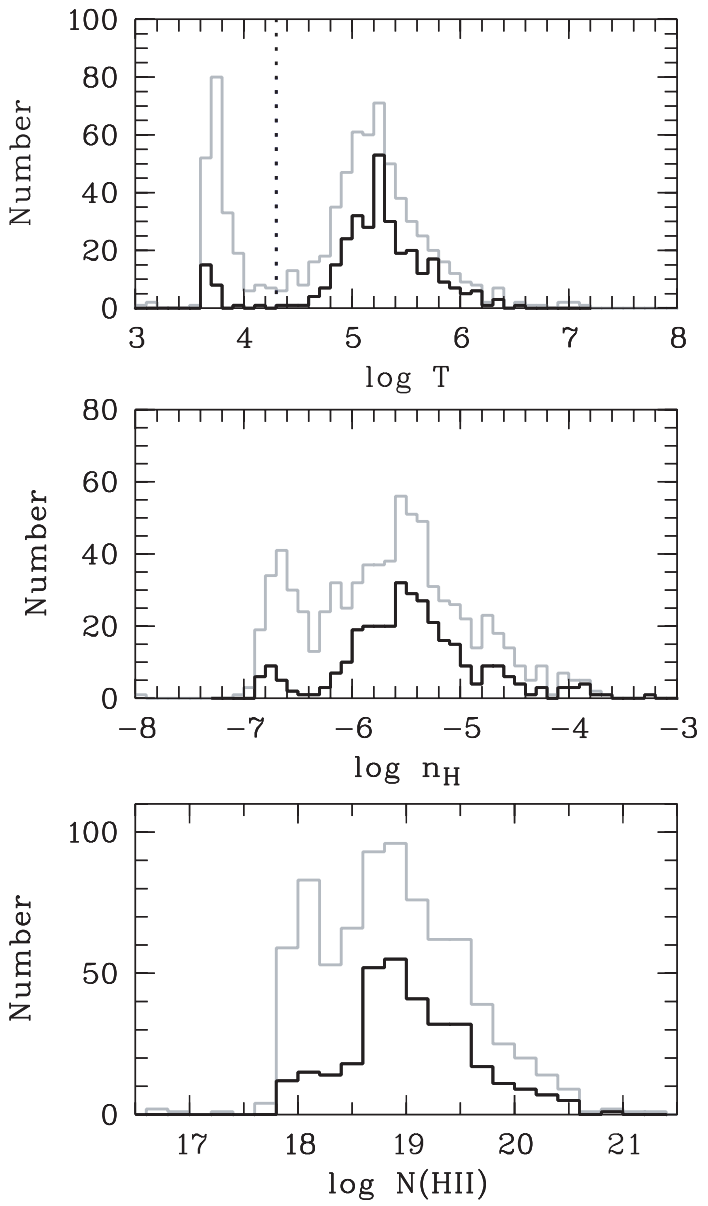

Fig. 4. Distribution of temperatures ( $T$ in $\mathrm{K}$ ), hydrogen volume densities $\left(n\right.$ in $\left.\mathrm{cm}^{-3}\right)$, and total hydrogen column densities $\left(N\right.$ in $\left.\mathrm{cm}^{-2}\right)$ for the total BLA sample (770 absorbers; shown in gray) and the high-quality sample (321 absorbers; shown in black).

Richter et al. 2006), so that the measured $b$ value of a BLA provides only an upper limit for the temperature of the gas. If we compare the $b$ value distribution of the total BLA sample in our simulation (lower panel in Fig. 2, gray line) with that of the highquality sample (black line), we see that the fraction of absorbers with $\log b<1.8\left(b<63 \mathrm{~km} \mathrm{~s}^{-1}\right)$ is very large in the total sample ( $\sim 70$ percent), while for the high-quality sample it is somewhat smaller ( $\sim 59$ percent). This implies that for low $b$ values the contamination from multi-component Ly $\alpha$ absorbers (i.e., systems for which $b_{\text {non-th }}$ is dominant) in the total BLA sample is substantial (see also Sects. 3.1, 4.1, and 5.1).

\section{Physical properties of BLAs}

\subsection{Temperature distribution}

We have determined for each BLA in our sample a characteristic temperature by calculating the mean of the temperatures in the cells that contribute to the $\mathrm{HI}$ Ly $\alpha$ absorption, weighted by $\mathrm{H} \mathrm{I}$ optical depth in each cell. The H I weighting is a crucial point, since it is the temperature in the cells with the largest H I optical depths that determines the thermal broadening (and thus the width) of the Ly $\alpha$ absorption. In Fig. 4, upper panel, we show the distribution of $\log T$ for the total sample (gray) and the highquality sample (black). The BLA temperature distribution is bimodal, with most BLAs spanning a range of $4.4 \leq \log T \leq 6.2$ with a peak near $\log T \approx 5.2$, thus typical for what is expected 

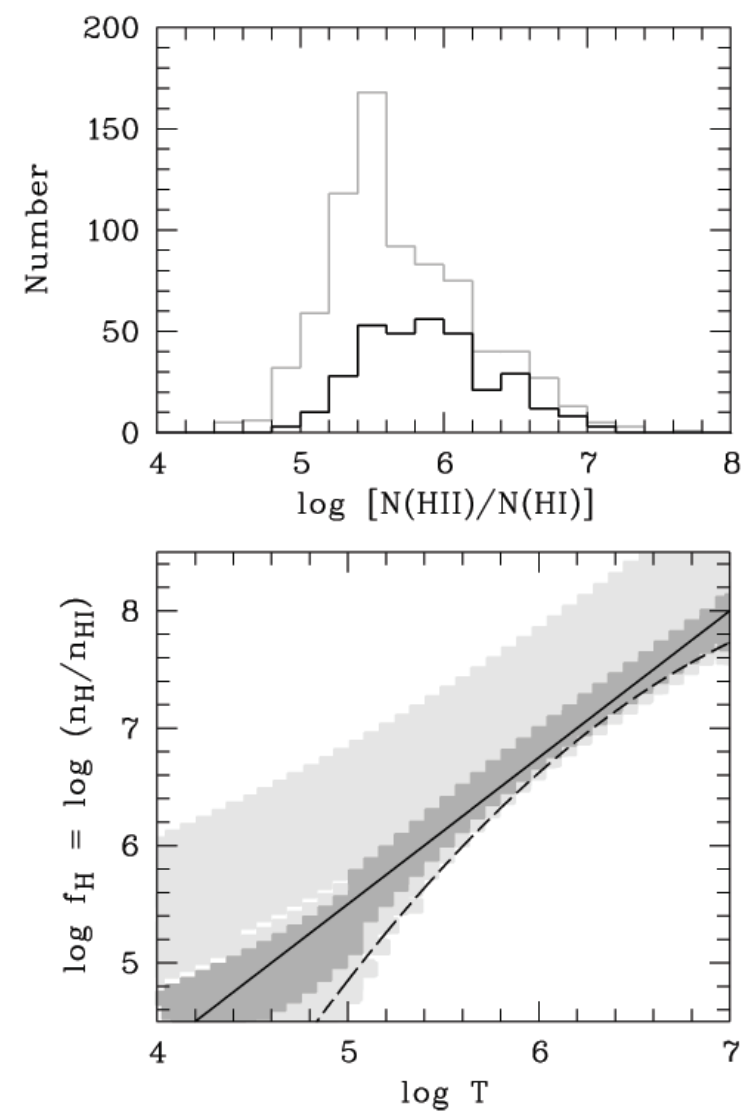

Fig. 5. Upper panel: distribution of ionization fractions in the total BLA sample (gray) and the high-quality sample (black). Lower panel: the hydrogen ionization fraction for each cell in our simulation (photoionization+collisional ionization), $\log f_{\mathrm{H}}=\log \left(n_{\mathrm{H}} / n_{\mathrm{HI}}\right)$, is plotted as a function of the gas temperature, $\log (T(\mathrm{~K}))$. The light gray shaded area indicates cells in the density range $\log n_{\mathrm{H}}=-5$ to -7 . The dark gray shaded area refers to cells that have $\log n_{\mathrm{H}}=-5.3$ to -5.6 , thus a density range that is characteristic for BLAs. The solid black line shows the best linear fit of the data in the BLA density range, the dashed black curve shows a function that approximates $\log f(T)$ for a pure collisional ionization model.

for the low-temperature WHIM (e.g., Davé et al. 2001). A second sharp peak is visible at lower temperatures near $\log T=3.8$, a temperature that is characteristic for the photoionized Ly $\alpha$ forest (e.g., Davé \& Tripp 2001). At such low temperatures, thermal broadening contributes only with $b_{\text {th }} \approx 10 \mathrm{~km} \mathrm{~s}^{-1}$ to the total line width of these systems (see Eq. (3)). The measured large width of these lines $\left(b \geq 40 \mathrm{~km} \mathrm{~s}^{-1}\right)$ therefore must be determined by non-thermal processes, such as unresolved component structure or macroscopic turbulence.

The BLA temperature distribution clearly separates lowtemperature, photoionized H I absorbers with $\log T<4.3$ (i.e., broad Ly $\alpha$ forest lines) from shock-heated, high-temperature WHIM absorbers that have $\log T \geq 4.3$ (indicated by the dotted line in Fig. 4, upper panel). While for the high-quality sample only about eight percent of the BLAs have temperatures $\log T<4.3$, the fraction of BLAs with $\log T<4.3$ in the total sample is $\sim 27$ percent. Nearly all (198 out of 209) of these lowtemperature BLAs in the total sample have $b$ values $\leq 63 \mathrm{~km} \mathrm{~s}^{-1}$ $(\log b \leq 1.8)$. This shows that BLA absorbers with $b$ values $<63 \mathrm{~km} \mathrm{~s}^{-1}$ in the total BLA sample are substantially contaminated by photoionized Ly $\alpha$ forest lines (see also Fig. 3 and Sect. 3.1), while for BLAs with $b \geq 63 \mathrm{~km} \mathrm{~s}^{-1}$ the collisionally ionized WHIM absorbers dominate the BLA population.
Note that due to the relatively small box size and the missing large-scale power in our simulation the characteristic temperatures of WHIM filaments and BLAs may be slightly underestimated. We are planning to examine this issue in more detail in a future paper using a higher-resolution simulation.

\subsection{Density distribution}

The distribution of logarithmic hydrogen volume densities $\left(n_{\mathrm{H}}\right.$ in units $\mathrm{cm}^{-3}$ ) in our two BLA samples is shown in Fig. 4, middle panel. In a similar way as for the temperature, we have calculated for each BLA $n_{\mathrm{H}}$ from the mean of the hydrogen volume densities in the cells that contribute to the $\mathrm{H}$ I absorption, weighted by the H I optical depth in each cell. The distribution of $\log n_{\mathrm{H}}$ spans a large range between -3.6 and -7.2 . Similar to the temperature distribution, the distribution of $\log n_{\mathrm{H}}$ is bimodal with one peak near $\log n_{\mathrm{H}} \approx-5.4$ and a second one near $\log n_{\mathrm{H}} \approx-6.7$. Again, this bimodal distribution separates the low-density, photoionized Ly $\alpha$ forest lines $\left(\log n_{\mathrm{H}}<-6.2\right.$, typically) from the higher-density WHIM absorbers ( $\log n_{\mathrm{H}} \geq-6.2$, typically). The above density peaks near $\log n_{\mathrm{H}}=-6.7$ and -5.4 correspond to overdensities of $\rho_{\mathrm{H}} / \overline{\rho_{\mathrm{H}}} \sim 1$ for the low-density Ly $\alpha$ forest and $\rho_{\mathrm{H}} / \overline{\rho_{\mathrm{H}}} \sim 20$ for the WHIM absorbers, thus similar to what has been found in other simulations (e.g., Davé \& Tripp 2001; Davé et al. 2001).

\subsection{Ionized hydrogen column densities}

The column density of ionized hydrogen in each BLA $(N(\mathrm{H}$ II $) \approx$ $N(\mathrm{H})$ ) can be estimated directly from our simulation, i.e., by integrating the hydrogen volume density, $n_{\mathrm{H}}$, over the cells that are located within the $2 \sigma$ velocity range of each BLA (see Eq. (1)). In the lower panel of Fig. 4 we show the distribution of $\log N(\mathrm{HII})$ for the total sample (gray) and the highquality sample (black). Most BLAs have ionized (=total) hydrogen column densities in the range $\log N(\mathrm{H}$ II $)=18-20$ with a peak near $\log N(\mathrm{HII})=18.8$. Less than seven percent of the BLAs have H II column densites larger than $10^{20} \mathrm{~cm}^{-2}$, but note that these systems contain a substantial fraction of the total gas column density in the BLAs ( $\sim 57$ percent in the total sample, $\sim 51$ percent in the high-quality sample). 87 percent of the broad Ly $\alpha$ forest lines with $\log T<4.3$ have H II column densities $\log N(\mathrm{H}$ II $)<18.5$. Broad absorbers that arise in the WHIM therefore dominate the distribution of the BLA H II column densities in the range $\log N(\mathrm{H}$ II $)=18.5-21.0$.

\subsection{Ionization fraction}

In the upper panel of Fig. 5 we have plotted the distribution of the ionized hydrogen ionization fractions, $N(\mathrm{HII}) / N(\mathrm{HI})$, for our two BLA samples. The logarithmic ionization fractions in both samples typically range between 5 and 7 with a peak near 5.6.

In the following we want to explore in more detail the individual ionization mechanisms (collisional ionization and photoionization) and their role in the observed $N(\mathrm{H} \mathrm{II}) / N(\mathrm{HI})$ distribution. For pure collisional ionization and in a collisional ionization equilibrium, the hydrogen ionization fraction, $\log f_{\mathrm{H}}=\log \left(n_{\mathrm{H}} / n_{\mathrm{HI}}\right)$, is governed solely by the gas temperature (e.g., Sutherland \& Dopita 1993). The ionization fraction then can be approximated via

$\log f_{\mathrm{H}}(T) \approx-13.9+5.4 \log T-0.33(\log T)^{2}$.

However, as the densities in the WHIM absorbers are low $\left(\log n_{\mathrm{H}}<-5\right.$, typically; see Sect.4.2), it is likely that 
photoionization contributes to the ionization fraction of the gas. To assess the temperature dependence of $f_{\mathrm{H}}$ in our combined collisional+photoionization model we have plotted in Fig. 5, lower panel, for each of the $\sim 1.5 \times 10^{6}$ cells along our 3000 LOS the ionization fraction, $\log f_{\mathrm{H}}$, as a function of the gas temperature, $\log T$. The light gray shaded area shows cells with hydrogen volume densities in the range $\log n_{\mathrm{H}}=-5$ to -7 . The dark gray shaded area shows cells with $\log n_{\mathrm{H}}=-5.3$ to -5.9 , thus in a density range that is characteristic for the shock-heated BLAs (see Fig. 3). The dashed black curve indicates the $f_{\mathrm{H}}-T$ relation for pure collisional ionzation (Eq. (4)). As clearly visible, all points lie above the relation for collisionally ionized gas, demonstrating that photoionization is important. For the density range that is typical for the BLAs in our simulation $\left(\log n_{\mathrm{H}}=-5.3\right.$ to -5.9 ; dark gray shaded area) the combined collisional+photoionization model suggests that $\log f_{\mathrm{H}}$ is roughly linearly related to $\log T$, so that

$\log f_{\mathrm{H}}(T) \approx-0.75+1.25 \log T$

This approximation is shown in Fig. 5, lower panel, as a solid black line.

\subsection{Non-thermal line broadening in BLAs}

In Fig. 6, upper panel, we have plotted the logarithmic $b$ values from the line fitting of our BLA sample against $\log b_{\text {th }}$, which represents the thermal contribution to $b$ for each BLA, as calculated from the BLA temperatures (Sect. 4.1) and the inversion of Eq. (3). The gray dots show the data points for our total BLA sample, the black dots show the data points for the high-quality sample. For pure thermal broadening, $b=b_{\text {th }}$ (Eq. (2)). This relation is shown as solid black line in the upper panel of Fig. 5. As expected from the BLA temperature distribution (Fig. 4), there are a number of low-temperature, photoionized systems that have $b_{\text {th }} \ll b$ because of non-thermal line broadening effects; these systems cluster near $\log b_{\text {th }}=1$. The BLAs that refer to the shock-heated WHIM roughly follow the slope of the relation $b=b_{\text {th }}$, but are systematically offset by a few percent. A statistical comparison between $b$ and $b_{\text {th }}$ for $\log b_{\text {th }} \geq 1.5$ implies that for both the total sample and the high-quality sample the relation $b=1.1 b_{\text {th }}$ gives the best fit to the data. This means that non-thermal broadening mechanisms contribute (on average) with $\sim 10$ percent to the total $b$ value of a WHIM BLA in our simulation. From Eq. (2) then follows that (on average) $b_{\text {non-th }} \approx 0.5 b_{\text {th }}$ for these systems. Note that there are a number of systems that have $b<b_{\text {th }}$. Although this appears nonsensical at first, an inspection of these systems suggests that their absorption profile is dominated by a cooler gas component that has a relatively large H I optical depth. The fitted $b$ value in these systems only reflects the temperature of this cooler gas component, but is not good measure for the temperature of the hot gas, whose absorption is hidden in a broad wing that is ignored by AutoVP. Although $b_{\text {th }}$ is based on the optical-depth weigthed temperature of the gas in our BLAs, $b<b_{\text {th }}$ can occur in a few systems where this effect is particularly pronounced.

\subsection{Indirect estimate of the ionized gas content in BLAs}

The combination of the Eqs. (2), (3), (5), and $b=1.1 b_{\text {th }}$ allows us to estimate the ionization fraction, $f_{\mathrm{H}}$, in a thermally broadened BLA indirectly from its measured $b$ value. Together with the measured H I column density one thus can estimate the
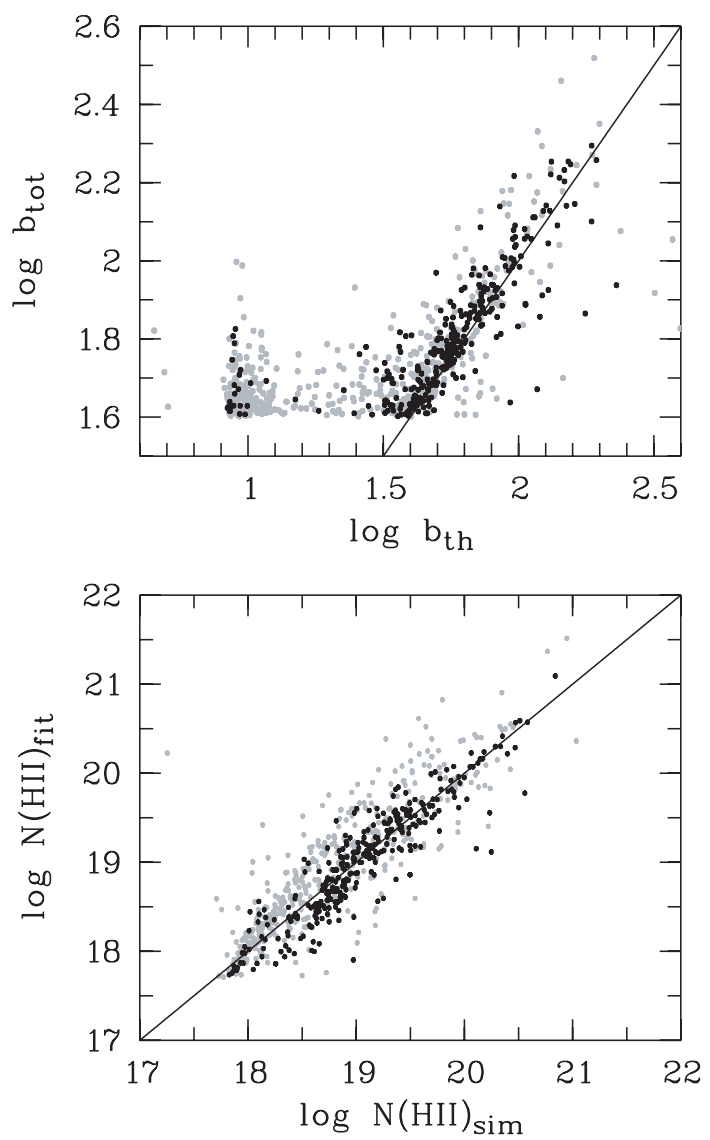

Fig. 6. Upper panel: the total $b$ values ( $b$ in units $\mathrm{km} \mathrm{s}^{-1}$ ) for the BLAs as obtained from the profile fitting are plotted against the thermal components, $b_{\text {th }}$, as calculated for each BLA from the H I optical depth weighted temperature in each system (Sect. 4.1). The solid black line indicates $b_{\mathrm{tot}}=b_{\mathrm{th}}$. Lower panel: comparison between the total hydrogen column densities ( $N$ in units $\mathrm{cm}^{-2}$ ) estimated indirectly from the measured line widths and H I column densities $\left(\log N\left(\mathrm{H} \mathrm{II}_{\text {fit }}\right.\right.$; see Sect. 4.6) and derived directly from the simulation $\left(\log N(\mathrm{H} \mathrm{III})_{\text {sim }}\right.$; Sect. 4.3). The black solid line indicates $\log N\left(\mathrm{H}_{\text {II }}\right)_{\text {fit }}=\log N\left(\mathrm{H}_{\text {II }}\right)_{\text {sim }}$.

total hydrogen column density in a BLA via $N(\mathrm{H}$ II $)=f_{\mathrm{H}} N(\mathrm{HI})$. Combining all these equations gives

$\log N(\mathrm{H}$ II $) \approx \log N(\mathrm{H} \mathrm{I})+1.25 \log \left(0.81 b^{2}\right)+1.47$.

Since $b$ and $N(\mathrm{HI})$ are the only parameters of BLAs that can be measured in real FUV spectra, this indirect estimate of $N(\mathrm{H} \mathrm{II})$ is the only possible method to observationally constrain the total gas budget and thus the baryon content of BLAs in the lowredshift Universe (see Richter et al. 2006). With our simulation we now want to examine the reliability of this indirect method for our sample of BLA absorbers. In Fig. 6, lower panel, we have plotted $N(\mathrm{H} \text { II })_{\text {fit }}$ against $N(\mathrm{HII})_{\text {sim }}$ for the total sample (gray dots) and the high-quality sample (black dots). $N(\mathrm{H} \mathrm{II})_{\mathrm{fit}}$ is the hydrogen column density derived indirectly via Eq. (6) and $N(\mathrm{H} \mathrm{II})_{\text {sim }}$ is the hydrogen column density obtained directly from the simulation using the cell integration (Sect. 4.3). The black solid line indicates the ideal case, $N(\mathrm{H} \mathrm{II})_{\text {fit }}=N(\mathrm{H} \mathrm{II})_{\text {sim }}$. Although the data points show a significant scatter around that line, the plot implies that (for a large enough sample) the indirect method indeed serves as a valuable tool to roughly estimate the total hydrogen column densities in BLAs. For the total sample the mean scatter is 0.25 dex, thus larger than for the restricted sample ( $0.18 \mathrm{dex})$ owing to the fact that non-thermal line 

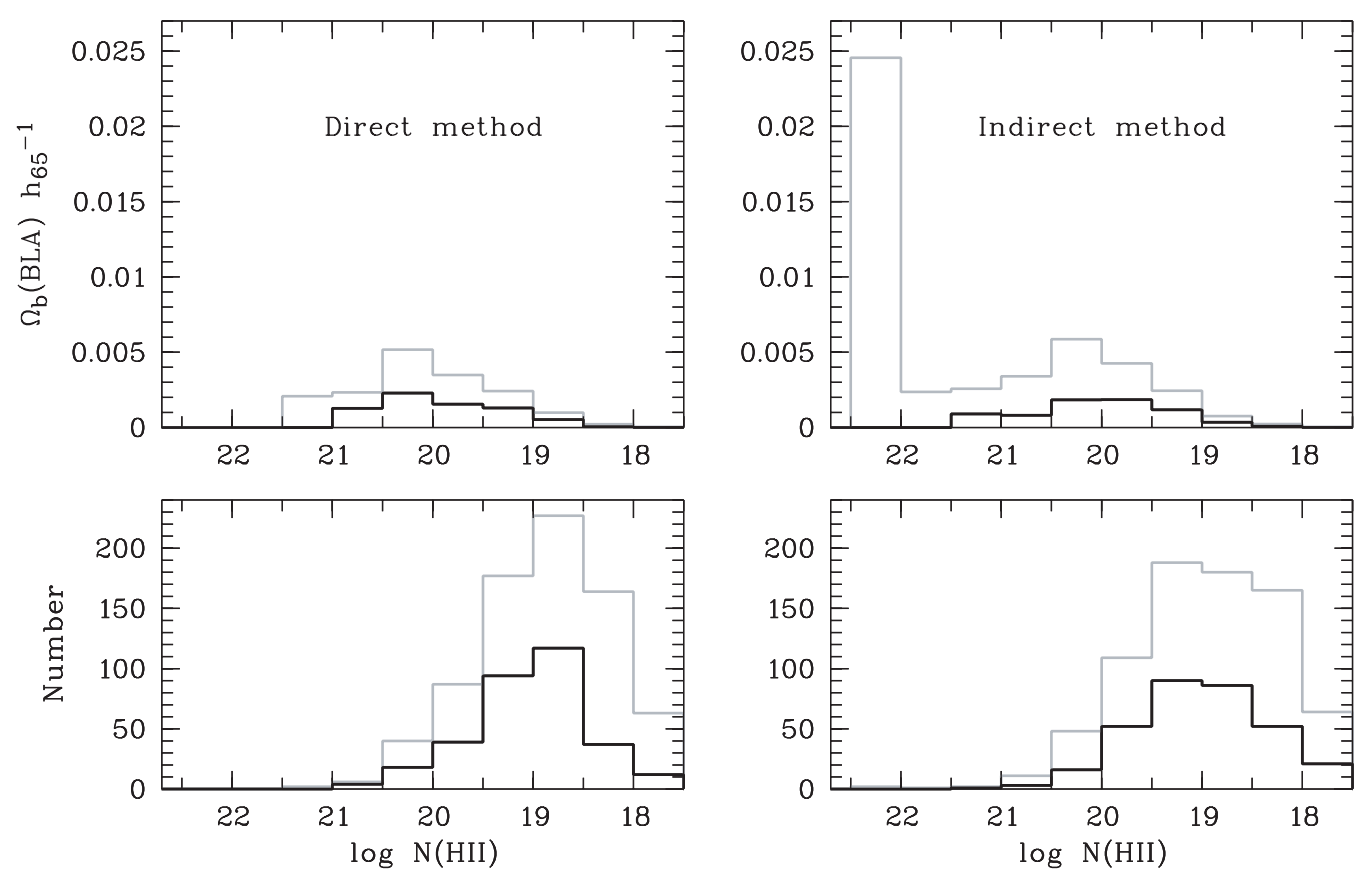

Fig. 7. Distribution of $\Omega_{\mathrm{b}}$ (BLA) and BLA numbers for bins of $\log N\left(\mathrm{H}\right.$ II), where $N$ is in units $\mathrm{cm}^{-2}$. The left panel shows the distribution based on the direct method to estimate $N(\mathrm{H}$ II) (cell integration), the right panel shows the distribution based on the indirect method (Eq. (6)). The bin size for $\log N(\mathrm{H}$ II $)$ is 0.5 dex.

broadening processes in these systems cause a considerable uncertainty for the indirect estimate of $N(\mathrm{H} \mathrm{II})$.

\section{The baryon content of BLAs}

\subsection{The baryon content of BLAs in the simulation}

From the cell integration (direct method; Sect. 4.3) we derive a total hydrogen column density of $2.31 \times 10^{22} \mathrm{~cm}^{-2}$ for the total BLA sample (770 systems) and $9.67 \times 10^{21} \mathrm{~cm}^{-2}$ for the highquality sample (321 systems). The cosmological mass density of the BLAs in terms of the current critical density $\rho_{\mathrm{c}}$ is given by

$\Omega_{\mathrm{b}}(\mathrm{BLA})=\frac{\mu m_{\mathrm{H}} H_{0}}{\rho_{\mathrm{c}} c} N(\mathrm{HII})_{\mathrm{total}} \Delta X^{-1}$,

where $\mu=1.3, m_{\mathrm{H}}=1.673 \times 10^{-27} \mathrm{~kg}, H_{0}=65 \mathrm{~km} \mathrm{~s}^{-1} \mathrm{Mpc}^{-1}$, and $\rho_{\mathrm{c}}=3 H_{0}{ }^{2} / 8 \pi G$. To compare our results with recent observations we assume at this point that the comoving path length for our BLA sample is $\Delta X=\Delta z=20.0$ (i.e., we assume that $\left.q_{0}=0\right)$. Using Eq. (7), the values for $N(\mathrm{H} \mathrm{II})_{\text {total }}$ listed above correspond to baryon densities of $\Omega_{\mathrm{b}}$ (BLA) $=0.0167 h_{65}{ }^{-1}$ and $0.0070 h_{65}{ }^{-1}$, respectively. From this follows that the BLAs trace a considerable fraction of the baryons in our simulation ( $\sim 35$ percent in the total sample, $\sim 15$ percent in the high-quality sample). Note that the contribution to $\Omega_{b}(B L A)$ from photoionized absorption systems with $\log T<4.3$ (Ly $\alpha$ forest) is negligible ( $\sim 2$ percent in the total sample and $<1$ percent in the highquality sample). In the upper left panel of Fig. 7 we show the distribution of baryons as a function of the total hydrogen column density for the direct method. The gray line refers to the total sample, the black line indicates the high-quality sample. Values for $\log N$ (H II) are given in 0.5 dex-wide bins and the values for $\Omega_{b}(B L A)$ indicate the baryon content per bin. The lower left panel of Fig. 7 shows the number of BLAs in each columndensity bin. From this comparison we learn that BLA systems with $\log N(\mathrm{H}$ II $)=19.5-20.5$ contribute most to $\Omega_{\mathrm{b}}(\mathrm{BLA})$
( $\sim 56$ percent), while they contribute with only $\sim 16$ percent to the total number of BLAs.

\subsection{The baryon content of BLAs derived from the indirect estimate}

Using the indirect method for the determination of $N(\mathrm{H} \mathrm{II})$ (Eq. (6)), we obtain values of $\Omega_{\mathrm{b}}$ (BLA) $=0.0465 h_{65}{ }^{-1}$ (total sample) and $0.0070 h_{65}^{-1}$ (high-quality sample). Thus, while direct and indirect method give identical results for the high-quality sample, the indirect method vastly overestimates $\Omega_{\mathrm{b}}$ (BLA) for the total sample. In the right panel of Fig. 7 we plot (in a way similar as for the direct method) the distribution of $\Omega_{\mathrm{b}}(\mathrm{BLA})$ and BLA numbers for bins of $\log N(\mathrm{H}$ II $)$. When compared to the left panel, it immediately becomes clear that the indirect method wrongly suggests the presence of a few (three) BLA systems with very large total hydrogen column densities $(\log N(\mathrm{H}$ II $)>21.5)$ that would contain more baryonic matter than all the other BLAs together. This is also seen in the lower panel of Fig. 6, where most of the few points with $\log N(\mathrm{H} \mathrm{II})_{\text {sim }} \geq 20.5$ clearly lie above the expected relation $N(\mathrm{H} \mathrm{II})_{\text {fit }}=N(\mathrm{HII})_{\text {sim }}$. A visual inspection of these BLA systems shows that all but one systems are multi-component absorbers whose widths are determined mostly by non-thermal line broadening. The velocity-component structure is not properly accounted for in the fit by AutoVP, resulting in large discrepancies between $N(\mathrm{H} \mathrm{II})_{\text {fit }}$ and $N(\mathrm{H} \mathrm{II})_{\text {sim }}$ for these systems. As these high-column density absorbers dominate $\Omega_{\mathrm{b}}$ (BLA), the overestimate of $N(\mathrm{H}$ II $)$ for these systems with the indirect method together with the low-number statistics in the high-column density regime introduces a substantial uncertainty for the (indirect) determination of $\Omega_{b}(B L A)$. The discrepancy in $\Omega_{b}$ (BLA) between the direct and indirect method becomes less severe if we ignore these few high-column density systems. If we restrict our total BLA sample to values of $\log N(\mathrm{H} \text { II })_{\text {fit }} \leq 20.5$ (754 systems), we derive values of $\Omega_{\mathrm{b}}(\mathrm{BLA})=0.0121 h_{65}{ }^{-1}$ (cell integration) and 
Table 1. Distribution of the baryons in BLAs with $\log \left(N\left(\mathrm{~cm}^{-2}\right) / b\left(\mathrm{~km} \mathrm{~s}^{-1}\right)\right) \geq 10.7$.

\begin{tabular}{lrrrr}
\hline \hline Selection & $\mathcal{N}$ & $\mathrm{d} \mathcal{N} / \mathrm{d} z$ & $\Omega_{\mathrm{b}}(\mathrm{BLA})_{\text {sim }} h_{65}$ & $\Omega_{\mathrm{b}}(\mathrm{BLA})_{\mathrm{fit}} h_{65}$ \\
\hline Total sample & 770 & 39 & 0.0167 & 0.0465 \\
High-quality sample & 321 & 16 & 0.0070 & 0.0070 \\
\hline$b=40-100 \mathrm{~km} \mathrm{~s}^{-1}$ & 700 & 35 & 0.0076 & 0.0102 \\
$b=100-200 \mathrm{~km} \mathrm{~s}^{-1}$ & 64 & 3 & 0.0064 & 0.0068 \\
$b>200 \mathrm{~km} \mathrm{~s}^{-1}$ & 6 & $<1$ & 0.0027 & 0.0295 \\
$b=60-200 \mathrm{~km} \mathrm{~s}^{-1}$ & 268 & 13 & 0.0104 & 0.0111 \\
\hline $\log N(\mathrm{H} \text { II })_{\text {fit }} \leq 20.5$ & 754 & 38 & 0.0121 & 0.0136 \\
$\log N(\mathrm{H} \mathrm{II})_{\text {fit }} \leq 20.0$ & 706 & 35 & 0.0076 & 0.0077 \\
$\log N(\mathrm{H} \text { II })_{\text {fit }} \leq 19.5$ & 597 & 30 & 0.0038 & 0.0035 \\
$\log N(\mathrm{H} \text { II })_{\text {fit }} \leq 19.0$ & 409 & 20 & 0.0012 & 0.0010 \\
\hline
\end{tabular}

$0.0136 h_{65}{ }^{-1}$ (indirect method), thus a much better agreement between these two methods. In Table 1 we compare values for $\Omega_{\mathrm{b}}$ (BLA) from the direct and the indirect method for a number of different selection criteria. From this follows that the agreement between direct and indirect method actually is quite good if one ignores the very broadest BLAs that have $b$ values larger than $200 \mathrm{~km} \mathrm{~s}^{-1}$.

Following the statistics in our simulation, the best range for the analysis of BLA systems in future high-resolution, high $S / N$ FUV spectra is $b=60-200 \mathrm{~km} \mathrm{~s}^{-1}$. For this range, our analysis yields $(\mathrm{d} \mathcal{N} / \mathrm{d} z)_{\mathrm{BLA}} \approx 13$ and values for $\Omega_{\mathrm{b}}(\mathrm{BLA})$ of $0.0104 h_{65}^{-1}$ (cell integration) and $0.0111 h_{65}^{-1}$ (indirect method). Thus, while the agreement between direct and indirect method is excellent for these $b$ values, the contamination of the BLA number density by photoionized Ly $\alpha$ forest lines with $\log T<4.3$ is negligible ( $\sim 5$ percent $)$.

\subsection{Comparison with FUV observations}

Richter et al. (2006) have studied properties and baryon content of BLAs in a sample of four low-redshift quasars using STIS high-resolution spectra. Along a total redshift path of $\Delta z=0.928$ they detect 49 BLA candidate systems above a sensitivity limit of $\log (N / b) \approx 11.3$. However, only 20 of these candidates with $b<100 \mathrm{~km} \mathrm{~s}^{-1}$ are considered as secure detections. From these 20 BLAs, Richter et al. (2006) obtain values of $(\mathrm{d} \mathcal{N} / \mathrm{d} z)_{\mathrm{BLA}}=22$ and $\Omega_{\mathrm{b}}(\mathrm{BLA})=0.0025 h_{65}{ }^{-1}$. It is important to note that their estimate of $\Omega_{b}(B L A)$ is based on the assumption that the gas is in collisional ionization equilibrium. Yet, our simulation shows that photoionization is important when it comes to the estimate of total hydrogen column densities and $\Omega_{\mathrm{b}}$ (BLA). If we take the 20 BLA candidates (secure detections) in the STIS data presented by Richter et al. (2006) and transform their measured $\mathrm{H}$ I column densities and $b$ values (their Table 4) into total gas column densities using Eq. (6) (i.e., now taking both collisional ionization and photoionization into account), we obtain a total hydrogen column density of $3.45 \times 10^{20} \mathrm{~cm}^{-2}$, corresponding to $\Omega_{\mathrm{b}}(\mathrm{BLA})=0.0047 h_{65}{ }^{-1}$. This estimate lies above the original estimate of $\Omega_{\mathrm{b}}(\mathrm{BLA})=0.0025 h_{65}{ }^{-1}$ by Richter et al., suggesting that the consideration of photoionization is important for the estimate of the baryon content of BLAs. If we restrict our total BLA sample in our simulation to $\log (N / b) \approx 11.3, \log N(\mathrm{H} \mathrm{II}) \leq 20.5$ and $b<100 \mathrm{~km} \mathrm{~s}^{-1}$, we obtain $(\mathrm{d} \mathcal{N} / \mathrm{d} z)_{\mathrm{BLA}}=18$ and $\Omega_{\mathrm{b}}(\mathrm{BLA})=0.0058 h_{65}{ }^{-1}$. Thus, despite the small-number statistics for BLAs in the STIS data, observations and simulations are in good agreement with each other.

Our analysis suggests that the study of BLAs in lowredshift quasar spectra represents a useful method to study the distribution and baryon content of the WHIM. However, a number of BLA candidates significantly larger than currently available is desired to constrain the properties of these absorbers on a statistically secure basis. For this, future FUV measurements of BLAs at high spectral resolution and sufficient $S / N$ will be required.

\section{The kinematic relation between BLAs and $\mathrm{O} \mathrm{VI}$ absorbers}

A considerable fraction of the BLAs in our sample shows associated O VI absorption within a velocity radius of $50 \mathrm{~km} \mathrm{~s}^{-1}$. Number distribution and equivalent-width distribution of the O VI absorbers are discussed in detail in Fang \& Bryan (2001). Here we focus on the kinematic relationship between broad Ly $\alpha$ absorption and associated O VI $\lambda 1031.9$ absorption in our simulation. Recent STIS observations of BLAs and associated O VI absorbers (Richter et al. 2006) show that many of the BLA/O VI absorber pairs have velocity offsets between the $\mathrm{HI}$ and the $\mathrm{O}$ VI absorption. In addition, measured $\mathrm{O}$ VI line widths often are inconsistent with those of the broad $\mathrm{H}$ I absorbers (if a single-component absorption is assumed). These measurements imply that sub-component structure is present and/or that the broad Ly $\alpha$ absorption and the O VI absorption do not trace the same gas phase. We now want to study these interesting aspects in our artificial spectra.

From the 770 BLA systems in our sample, 274 systems have O VI absorption that is accurately fitted by AutoVP. Note that many of these O VI absorbers are composed of several subcomponents. For this analysis we consider only the dominating $\mathrm{O}$ VI sub-component for each BLA with the highest O VI optical depth. In Fig. 8, upper panel, we have plotted the distribution of the velocity offsets between the line centroids of the $\mathrm{HI}$ and the O VI absorption, $\Delta v=\left|v_{\mathrm{HI}}-v_{\mathrm{OVI}}\right|$. O VI absorbers that refer to BLAs from the total sample (274 systems) are plotted in gray, O VI systems that refer to BLAs in the high-quality sample (145 systems) are plotted in black. For the total sample the median offset between HI and O VI is $6.3 \mathrm{~km} \mathrm{~s}^{-1}$, while for the high-quality sample the median offset is $5.3 \mathrm{~km} \mathrm{~s}^{-1}$. This implies that small velocity offsets between the H I Ly $\alpha$ absorption and the O VI absorption are common. A possible explanation for such velocity offsets is that some of the gas filaments that give rise to broad H I and O VI absorption have a relatively complex (non-Gaussian) density and temperature distribution (see, e.g., Fig. 1, right panel). While the BLAs trace the H I optical-depth weighted density distribution of the filaments at all temperatures, O VI absorbers preferentially arise from higher-density regions in a small temperature range around $3 \times 10^{5} \mathrm{~K}$. Therefore, BLAs and O VI absorbers most likely originate in somewhat 

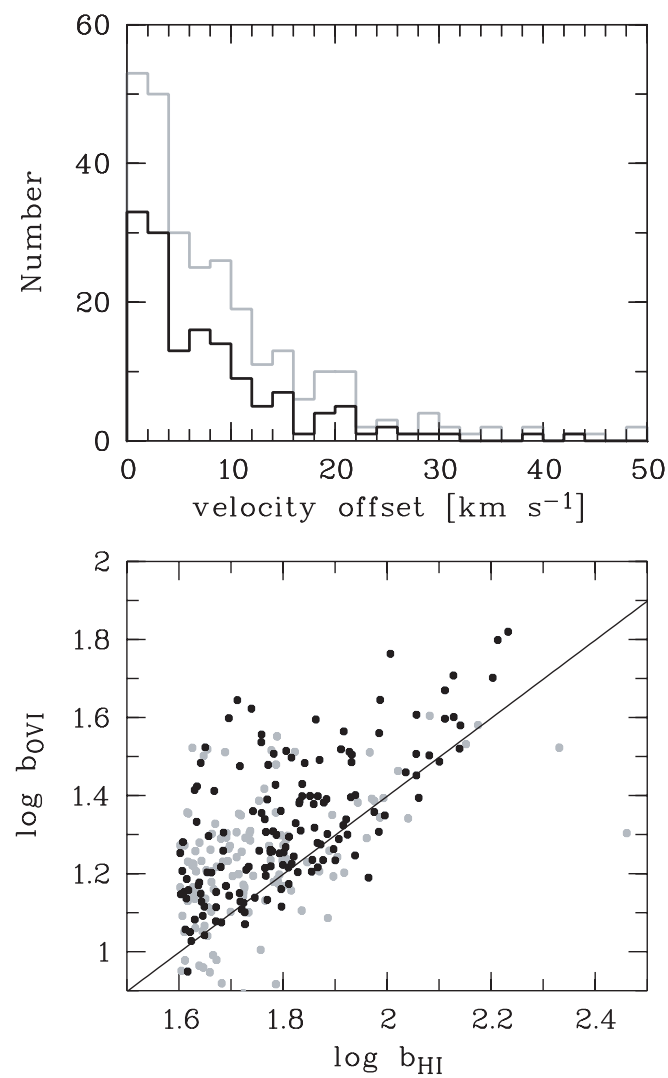

Fig. 8. Upper panel: the distribution of velocity offsets between broad Ly $\alpha$ absorption and associated O VI absorption is shown (gray line $=$ total sample; black line $=$ high-quality sample). Lower panel: O vI $b$ values are plotted against $\mathrm{HI} b$ values $\left(b\right.$ in units $\left.\mathrm{km} \mathrm{s}^{-1}\right)$. The solid line indicates $b(\mathrm{O} \mathrm{VI})=b(\mathrm{HI}) / 4$, as expected for pure thermal broadening (gray dots $=$ total sample; black dots $=$ high-quality sample).

different gas phases and regions within a filament. This would naturally explain the observed small velocity offsets between these two species in both simulations and observations.

In the lower panel of Fig. 8 we compare $\mathrm{HI}$ and $\mathrm{O}$ VI $b$ values for the total sample (gray dots) and the high-quality sample (black dots). If H I and O VI would trace the same gas phase of a purely thermally broadened WHIM absorber one would expect that $b(\mathrm{HI})=4 b(\mathrm{O} \mathrm{VI})$ owing to the 16 times larger weight of oxygen (see Eq. (2)). Most of the BLA/O VI absorber pairs lie above this expected relation (in Fig. 8, lower panel, shown as solid line) and the data points show a large scatter for both the total and the high-quality sample. The plot indicates that the $b$ values of H I and O VI are only very poorly related to each other. This is not surprising in view of the fact that for most BLAs the absorption of H I and O VI is offset by a few $\mathrm{km} \mathrm{s}^{-1}$ (see above). The O VI absorbers have widths significantly larger than what is expected for pure thermal broadening in these systems, suggesting that most of the $\mathrm{O}$ VI $b$ values are determined predominantly by non-thermal processes.

\section{Summary}

In this paper we have used artificial spectra generated from a hydrodynamical simulation to study frequency, distribution, physical conditions, and baryon content of broad Ly $\alpha$ absorbers (BLAs) in the low-redshift intergalactic medium. Along a total redshift path of $\Delta z=20$ we find 770 BLA systems with $b \geq 40 \mathrm{~km} \mathrm{~s}^{-1}$ above a detection threshold of $\log \left(N\left(\mathrm{~cm}^{-2}\right) / b\left(\mathrm{~km} \mathrm{~s}^{-1}\right)\right) \geq 10.7$, implying a number density of BLA systems per unit redshift of $(\mathrm{dN} / \mathrm{d} z)_{\mathrm{BLA}}=39$ for this sensitivity limit. From a spectral analysis with automatted Voigtprofile fitting we find typical H I column densities of the BLAs of $\log N(\mathrm{HI}) \approx 13$ and typical $b$ values of $40-100 \mathrm{~km} \mathrm{~s}^{-1}$. As a visual inspection suggests, only 321 of these BLAs are single-component, Gaussian shaped absorbers, while the remaining 449 systems show evidence for sub-component structure and multiple unresolved velocity components. Our simulation implies that BLAs predominantly trace shock-heated gas in the warm-hot intergalactic medium (WHIM) in the temperature range $\log T=4.4-6.2$. However, photoionized systems at lower temperatures $(\log T<4.3)$ also contribute to the population of BLAs ( $\sim 27$ percent by number). BLAs that belong to the WHIM typically have overdensities of 20 and characteristic ionization fractions of $\log f_{\mathrm{H}} \approx 5.6$. At these densities, both collisional ionization and photoionization contribute to the total ionization fraction in the gas. We find that in the characteristic BLA density range $\log f_{\mathrm{H}}$ scales linearly with $\log T$, so that for pure thermal line broadening the ionization fraction can be estimated from the BLA line width. The total hydrogen column densities of the BLAs typically lie in the range $\log N(\mathrm{H} \mathrm{II})=18-20$ with a peak near $\log N(\mathrm{H}$ II $)=18.8$. Most of the photoionized systems have logarithmic total hydrogen column densities $<18.5$ and thus do not contribute significantly to the the total baryon content of BLAs. We find that non-thermal line broadening mechanisms contribute (on average) with $\sim 10$ percent to the total $b$ value of a WHIM BLA, suggesting that for most systems the measured line width represents a good indicator for the thermal state of the absorbing gas. Therefore, our simulation suggests that it is possible to estimate the total gas column density in BLAs from the measured $b$ values and H I column densities and to estimate the baryon content of these systems in observational data. Our data imply that this indirect method works well for systems with total logarithmic hydrogen column densities $<20.5$ and for a BLA sample that is sufficiently large. The total baryon content of all BLAs in our simulation in the range $\log N(\mathrm{H}$ II $) \leq 20.5$, $b \geq 40 \mathrm{~km} \mathrm{~s}^{-1}$ and $\log (N / b) \geq 10.7$ (754 systems; $\mathrm{d} \mathcal{N} / \mathrm{d} z \approx 38$ ) is $\Omega_{\mathrm{b}}(\mathrm{BLA})=0.0121 h_{65}{ }^{-1}$, which represents $\sim 25$ percent of the total baryon budget in our simulation. This result supports the idea that BLAs represent a significant baryon reservoir at low redshift. If we restrict our total BLA sample to the range $\log N(\mathrm{H} \mathrm{II}) \leq 20.5, b=40-100 \mathrm{~km} \mathrm{~s}^{-1}$ and $\log (N / b) \geq 11.3$, we obtain $(\mathrm{d} \mathcal{N} / \mathrm{d} z)_{\mathrm{BLA}} \approx 18$ and $\Omega_{\mathrm{b}}(\mathrm{BLA})=0.0058 h_{65}{ }^{-1}$, in agreement with recent FUV observations.

Our simulation indicates that future observational studies of BLAs at low $z$ will be of great importance to study the baryon content of the WHIM, but for a reliable estimate of $\Omega_{b}(B L A)$ a large sample $(n>10)$ of high-resolution, high $S / N$ FUV spectra of low redshift QSOs will be required. The Cosmic Origin Spectrograph (COS), originally scheduled to be implemented on the Hubble Space Telescope (HST) in 2004, would have been the ideal instrument for these observations, but in view of the uncertain status of the COS mission and other future FUV instruments it remains unclear whether such data will be available in the near future. Next to observations, additional simulations of the lowredshift IGM with higher resolution and more realistic physics (e.g., galaxy feedback, metal-line cooling, non-equilibrium processes, etc.) will be important to investigate the physical conditions in BLAs in more detail and to re-examine the results presented in this paper.

Acknowledgements. P.R. acknowledges financial support by the German Deutsche Forschungsgemeinschaft, DFG, through Emmy-Noether grant Ri 1124/3-1. T.F. is supported by the NASA through Chandra Postdoctoral Fellowship Award Number PF3-40030 issued by 
the Chandra X-ray Observatory Center, which is operated by the Smithsonian Astrophysical Observatory for and on behalf of the NASA under contract NAS 8-39073. G.L.B. is partially supported by NSF grant AST0507161. We thank Chris McKee for helpful comments.

\section{References}

Bryan, G. 1996, Ph.D. Thesis, Univ. Illinois, Urbana-Champaign Cen, R., \& Ostriker, J. 1999, ApJ, 514, 1

Danforth, C. W., \& Shull, J. M. 2005, ApJ, 624, 555

Davé, R., Hernquist, L., Weinberg, D. H., \& Katz, N. 1997, ApJ, 477, 21

Davé, R., Cen, R., Ostriker, J., et al. 2001, ApJ, 552, 473

Davé, R., \& Tripp, T. M. 2001, ApJ, 553, 528

Eisenstein, D. J., \& Hu, W. 1998, ApJ, 498, 137

Fang, T., \& Bryan, G. L. 2001, ApJ, 561, L31
Fang, T., Marshall, H. L., Lee, J. C., Davis, D. S., \& Canizares, C. R. 2002, ApJ, 572, L127 Ferland, G. J., Korista, K. T., Verner, D. A., et al. 1998, PASP, 110, 761 Haardt, F., \& Madau, P. 1996, ApJ, 461, 20

Nicastro, F., Mathur, S., Elvis, M., et al. 2005, Nature, 433, 495

Norman, M. L., \& Bryan, G. 1999, in Numerical Astrophysics, ed. S. M. Miyama, K. Tomisaka, \& T. Hanawa (Dordrecht: Kluwer), ASSL, 240, 19

Richter, P., Savage, B. D., Tripp, T. M., \& Sembach, K. R. 2004, ApJS, 153, 165 Richter, P., Savage, B. D., Sembach, K. R., \& Tripp, T. M. 2006, A\&A, 445, 827 Savage, B. D., Sembach, K. R., Tripp, T. M., \& Richter, P. 2002, ApJ, 564, 631 Sembach, K. R., Tripp, T. M., Savage, B. D., \& Richter, P. 2004, ApJS, 155, 351 Sutherland, R. S., \& Dopita, M. A. 1993, ApJS, 88, 253

Tripp, T. M., Savage, B. D., \& Jenkins, E. B. 2000, ApJ, 534, L

Valageas, P., Schaeffer, R., \& Silk, J. 2002, A\&A, 388, 741

Williger, G. M., Heap, S. R., Weymann, R. J., et al. 2006, ApJ, 636, 631

Zhang, Y., Anninos, P., Norman, M. L., \& Meiksin, A. 1997, ApJ, 485, 496 